\title{
Le blog de politicien : un espace de subjectivité
} affichée?

The Politician's Blog: A Space for Displaying Subjectivity?

Lotta Lehti

\section{OpenEdition}

Journals

Édition électronique

URL : http://journals.openedition.org/aad/1391

DOI : 10.4000/aad.1391

ISSN : $1565-8961$

Éditeur

Université de Tel-Aviv

Référence électronique

Lotta Lehti, "Le blog de politicien : un espace de subjectivité affichée? ", Argumentation et Analyse du Discours [En ligne], 9 | 2012, mis en ligne le 15 octobre 2012, consulté le 23 septembre 2019. URL http://journals.openedition.org/aad/1391 ; DOI : 10.4000/aad.1391

Ce document a été généré automatiquement le 23 septembre 2019

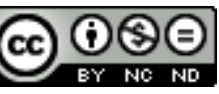

Argumentation \& analyse du discours est mis à disposition selon les termes de la licence Creative Commons Attribution - Pas d'Utilisation Commerciale - Pas de Modification 4.0 International. 


\title{
Le blog de politicien : un espace de subjectivité affichée?
}

The Politician's Blog: A Space for Displaying Subjectivity?

\author{
Lotta Lehti
}

\section{Introduction}

1 L'écriture de nombreux types et genres de discours se caractérise par un certain degré de subjectivité. Ainsi, pour ne prendre que ces deux exemples, la polémique suppose une prise de position forte et explicite (Kerbrat-Orecchioni 1997 [1980] : 170), alors que certains genres journalistiques sont traditionnellement considérés comme neutres et impartiaux (Koren 2004). Or, l'émergence des nouvelles technologies et des possibilités de publication instantanée et sans intermédiaires a bouleversé les enjeux de l'exposition et du gommage de la subjectivité du sujet. Koren (2009) nomme ce phénomène "subjectivité décomplexée ", expression qui renvoie à l'exposition franche et ouverte de la prise de position du sujet parlant, faisant partie aujourd'hui des conventions de plusieurs genres numériques. En parlant en particulier des blogs, Miller et Shepherd (2004) les considèrent comme le lieu d'un « art de soi » (art of the self) qui permet d'exposer le privé sur un forum public.

2 Le présent article s'intéresse à un genre particulier, à savoir le blog de politicien, avec l'objectif de voir dans quelle mesure il s'agit d'un espace de subjectivité affichée. Pour ce faire, nous examinerons quels sont les modes et le degré de la subjectivité dans ce genre, en mettant l'accent sur l'inscription de la subjectivité dans le langage et sur l'impact de son degré d'intensité. L'étude se concentre sur les billets postés par les politiciens auteurs de blog et laisse de côté les commentaires des lecteurs.

3 La notion de genre de discours sera entendue ici comme une pratique sociale, un prototype de situation communicative récurrente (Miller 1984). De ce point de vue, toutes les situations communicatives qu'accueille le format blog ne peuvent guère être décrites par un seul prototype ${ }^{1}$, car le blog est avant tout un format, accessible par le médium internet. Au sein de ce format, il est possible de repérer des situations 
communicatives récurrentes, c'est-à-dire des genres, parmi lesquels le blog de mode, le blog d'événement et le blog de politicien, auquel nous nous intéressons dans le présent article. Opérant sur un niveau plus général, Miller et Shepherd (2009) distinguent deux genres majeurs au sein du blog: le blog personnel (personal blog) et le blog d'affaires publiques (public affairs blog). Ils caractérisent le premier par l'exposition de la vie privée en public et le désir de construire une image de soi, alors que le but des blogs de la deuxième catégorie est l'action et le changement social (ibid.). Le blog de politicien, que nous distinguons d'ailleurs du «blog politique » renvoyant à tout blog traitant de thèmes politiques mais écrits par des citoyens, contient des éléments tantôt du blog personnel, tantôt du blog d'affaires publiques. Comme l'ont indiqué notamment Janoschka (2010), Suomela-Salmi (2009) et Jereczek-Lipińska (2007), l'un de ses objectifs principaux est la construction d'une image crédible de l'auteur. De plus, les sujets abordés dans les blogs des politiciens sont souvent, mais non exclusivement, liés à l'auteur et à ses activités ou opinions. Les blogs des politiciens contiennent pourtant également des éléments qui renvoient au blog d'affaires publiques selon la division de Miller et Shepherd (2009). En effet, dans certains blogs ou billets de blog, le politicien milite ouvertement en faveur ou contre une cause politique ou sociale. Cependant, l'objectif de projeter une image de soi ne naît guère de motifs exhibitionnistes, comme c'est le cas dans un blog personnel : il s'agit plutôt de persuader les électeurs de la crédibilité de la personne politique. La construction de l'image de soi participe du travail et de la campagne politiques, ce qui relève du blog d'affaires publiques.

L'inscription de la subjectivité dans le langage des blogs a été, à notre connaissance, peu étudiée. Parmi ces travaux, nous pouvons mentionner celui de Herring et Paolillo (2006), qui constatent une augmentation de l'usage des pronoms de la première personne dans les blogs « journaux extimes » (diary blogs) en comparaison avec les blogs «filtres» (filter blogs) consistant en des listes d'hyperliens. Myers (2010: 95-128) explore les voies de la subjectivité (stance) dans divers blogs, se concentrant sur l'expression et la formulation des opinions. Quant aux blogs des politiciens, Janoschka (2010) les examine du point de vue de la politesse linguistique et du marketing politique, considérant la subjectivité exprimée par exemple par l'usage des pronoms personnels comme une stratégie persuasive, sans entrer dans le détail du degré d'expression de la subjectivité.

\section{De la subjectivité dans le langage}

5 La subjectivité est ici comprise dans un sens large, tel que défini par Hancil (2011:7) : il s'agit de l' «expression, explicite ou implicite, des émotions et attitudes de l'énonciateur dans la construction et la cohésion discursives ». Kerbrat-Orecchioni (1997 [1980] : 157), à qui nous avons emprunté le titre de cette section, souligne l'omniprésence et la nature graduelle de l'expression de la subjectivité :

[T]oute séquence discursive porte la marque de son énonciateur, mais selon des modes et des degrés divers. La seule attitude légitime, c'est d'admettre que toute séquence se localise quelque part sur l'axe qui relie les deux pôles infiniment éloignés de l'objectivité et de la subjectivité ; la seule entreprise rentable, c'est d'essayer d'en identifier, différencier et graduer les divers modes de manifestation.

6 C'est cette démarche que nous adoptons dans la présente étude. Nous allons ainsi examiner si les billets des blogs de politiciens manifestent une subjectivité élevée, voire intense, ou si la subjectivité exprimée est d'un degré moindre. De plus, nous verrons 
comment la subjectivité est inscrite dans le discours. Nous esquissons un continuum allant du pôle subjectif (c'est-à-dire du langage «dans lequel l'énonciateur s'avoue explicitement " (Kerbrat-Orecchioni 1997 [1980] : 71) vers le pôle objectif (celui du langage «qui s'efforce de gommer toute trace de l'existence d'un énonciateur individuel » (ibid.).

7 Le principe sous-jacent de l'étude est le postulat de la linguistique énonciative (Benveniste 1966, Kerbrat-Orecchioni 1997 [1980]) selon lequel tout langage est subjectif car il porte des traces de l'énonciateur. Sur le plan plus pratique, l'article étudie la subjectivité dans le sens de la prise de position telle qu'elle est définie par Amossy et Koren (2004) : il s'agit de « s'impliquer personnellement sur une question qui autorise des réponses différentes, quand elle ne suscite pas des différends ». A la différence de la notion d'engagement, cependant, la prise de position «ne milite pas nécessairement pour une cause, ni ne tente de changer la face des choses » en ce qui concerne une situation sociale ou politique donnée. Ainsi, la prise de position se réfère à toute mise en mots des positionnements, des façons de voir et des questionnements du sujet parlant, alors que l'engagement renvoie à « une volonté délibérée et affichée de militer pour une cause et contribuer à effectuer des modifications de fait dans une situation sociale et politique donnée » (ibid.). Comme toute forme de subjectivité, la prise de position est graduée et peut se manifester à des degrés d'intensité différents.

8 Selon Charaudeau (1992: 648), le sujet parlant exprime son point de vue par rapport à trois objets : «à l'interlocuteur, à ce qu'il dit, et à ce que dit l'autre » et cela s'effectue dans le langage par l'organisation des catégories de la langue. Dans ce triple positionnement de l'énonciateur, la référence à l'interlocuteur et aux paroles des autres fait surgir l'intersubjectivité, qui est une dimension essentielle de la subjectivité: celle de la constante adaptation à autrui. Benveniste $(1966: 260)$ constate que la subjectivité se trouve dans une réalité dialectique englobant le «moi » et l'« autre » dans laquelle ces deux participants sont définis par leur relation mutuelle.

Comme nous l'avons mentionné dans l'introduction, les genres imposent souvent des conventions, tacites ou explicites quant à l'intensité de l'expression de la subjectivité. Selon Amossy et Koren (2004), « certains genres plus que d'autres autorisent le locuteur à s'engager personnellement; d'autres le contraignent, sous peine de violation de contrat, à s'interdire toute intervention, voire à dissimuler les traces de sa présence ». Parallèlement, en ce qui concerne l'affichage des émotions, Plantin (2011 : 191) note que « certains genres discursifs exigent le contrôle et la répression des émotions ; dans d'autres au contraire, leur exhibition est obligatoire ». Par exemple, les genres de la presse écrite peuvent être situés dans l'espace soit de l'information, soit de l'opinion, selon le degré de subjectivité exprimée (cf. notamment Adam 1997, Grosse 2001, Herman et Jufer 2001). Les conventions génériques tacites n'éliminent pourtant pas l'omniprésence de la subjectivité. Ainsi, par exemple, Cohen-Wiesenfeld (2004) a montré que même dans le discours diplomatique, fortement caractérisé par l'objectivité, le langage contient des marqueurs de la position prise de l'auteur. Koren (2004) remarque que le masque d'objectivité qu'adoptent les journalistes les empêche de voir « l'idéologie socialement approuvée » qu'ils défendent dans leur écriture, qu'ils conçoivent comme impartiale. 


\section{Corpus et méthode}

10 Le corpus de l'étude est constitué de 874 billets de blogs, soit les billets postés dans 80 blogs de politiciens français pendant le mois de septembre 2007. Cette période a été choisie au hasard mais avec l'objectif d'examiner les blogs en dehors d'une période de campagne électorale. Les politiciens auteurs de ces blogs représentent des partis différents (PS, UMP, Verts, MoDem, FN, PRG) et des fonctions différentes (conseillers municipaux, généraux et régionaux, maires, députés, sénateurs, ministres, députés européens). Le corpus représente la totalité des blogs de politiciens que nous avons trouvés par la démarche suivante: d'abord, deux sites (qui n'existent plus) nous ont fourni les adresses des blogs des socialistes et, respectivement, des conservateurs. Nous avons également fait une recherche Google avec les noms des députés (selon la liste du site de l'Assemblée Nationale) accompagnés par des mots tels que "blog », «blogue », « weblog », « journal » et « carnet ». Finalement, les blogs repérés nous ont donné accès à tout un réseau de blogs de politiciens à travers les listes des sections permanentes, intitulées par exemple «blogs d'amis » ou «blogs militants». Les blogs inclus dans le corpus répondent aux critères suivants :

1. le blog inclut une section de commentaires et des archives maniables;

2. l'auteur du blog est le politicien lui-même (au moins en apparence);

3. l'auteur est un politicien élu pour une fonction; par conséquent, les blogs des membres de parti, partisans, électeurs ou assistants parlementaires, par exemple, ne sont pas inclus.

Dans l'analyse des exemples tirés du corpus, nous respectons l'orthographe d'origine, y compris les déviations de la norme.

Ce même corpus a été employé dans une catégorisation des blogs de politicien en sousgenres en fonction de la scénographie choisie dans les différents types de billets, repérée à travers les critères de la théorie du genre: l'usage du format, le but communicatif, les rôles des participants et l'organisation textuelle (Lehti 2011). La typologie élaborée comprend cinq sous-genres, à savoir le journal extime (diary), la vitrine (scrapbook), le tableau d'affichage (notice-board), la réflexion (essay) et la polémique (polemic). Ces cinq types représentent les pratiques communicatives suivantes: premièrement, le journal extime sert surtout à rendre le travail du politicien plus transparent par la présentation des tâches du politicien et du déroulement de sa journée de travail. Tisseron (2011: 84) définit l'extimité comme «le processus par lequel des fragments du soi intime sont proposés au regard d'autrui afin d'être validés». Dans notre corpus, l'extimité porte sur l'exposition de la vie de la personne politique en public, y compris les tâches professionnelles. Quant au blog vitrine, il sert, lui aussi, à accroître la transparence, mais également à informer les lecteurs de sujets politiques variés : un blog vitrine est une plateforme de publication de documents parus ailleurs, par exemple de communiqués de presse, discours, articles de presse et interviews. Un tableau d'affichage est un sous-genre d'annonces courtes présentées pêle-mêle, avec deux tendances majeures: ce type englobe d'une part les invitations à l'action - répondre aux questions, participer à un événement ou acquérir un produit, entre autres - et d'autre part des faits divers portant sur une large gamme de sujets. Les deux derniers types, la réflexion et la polémique, traitent tous les deux de questions politiques et se différencient par leur mode d'argumentation et par leur style : contrairement à la réflexion, qui présente une question sans prise de position 
intense, la polémique est marquée par une opposition de thèses antagonistes et un ton qui s'apparente à la violence verbale.

La présente analyse de la prise de position dans le genre du blog de politicien est effectuée dans le sillage de cette typologie. A défaut de pouvoir analyser en détail le corpus entier, nous décrirons le degré de subjectivité investi dans chaque sous-genre à travers des exemples prototypiques. Notre méthode mobilise des critères aussi bien qualitatifs (marqueurs de subjectivité) que quantitatifs (degré d'intensité), combinaison qui n'est pas anodine. L'accent est pourtant mis sur les critères qualitatifs et la catégorisation quantitative est loin d'imposer des clivages exacts entre les classes. L'identification des marqueurs de subjectivité et de leur intensité est effectuée à travers une interprétation globale du discours plutôt que par l'application de critères préétablis. Dans l'analyse qui suit, nous donnons pourtant la priorité au niveau lexical. Il va de soi que l'évaluation globale de la prise de position ne peut se faire isolément du propos référentiel ; les traits langagiers seront analysés en interaction avec le sujet que traite le billet. C'est sous ces auspices que l'analyse qui suit présentera, à travers huit exemples, une répartition des sous-genres - journal extime, tableau d'affichage, vitrine, réflexion et polémique - en trois catégories selon le degré de subjectivité investi : forte, intermédiaire et faible. Le sous-genre du journal extime manifeste des degrés nettement variables de prise de position; il est par conséquent présenté dans deux catégories d'intensité.

\section{La prise de position des politiciens auteurs des blogs}

\section{Subjectivité forte}

Comme nous l'avons déjà mentionné dans la section 2, la notion de prise de position ne délimite pas les sujets face auxquels la position est prise. Dans les cas où la prise de position est intense, deux tendances principales ont pourtant été repérées dans le corpus. D'une part, certains politiciens expriment une prise de position intense en traitant de leurs propres expériences et activités. D'autre part, une prise de position intense est souvent exprimée par rapport aux thèmes politiques, ce qui fait d'ailleurs émerger un engagement dans le sens du terme défini dans la section 2. La première tendance réfère au sous-genre du journal extime et la seconde à celui de la polémique. Ces deux sous-genres sont respectivement illustrés par les exemples (1) et (2).

Dans l'exemple (1), la prise de position intense est exprimée par une affectivité et une émotivité élevées. Il s'agit d'un billet tiré du blog du conseiller régional de l'île de France Jean-Luc Romero (UMP, http://www.romero-blog.fr/) :

(1) (numéros de ligne et italiques rajoutés, caractères gras et soulignement comme dans l'original)

1. Sheila à l'olympia

2. Après cette dure semaine à suivre le chemin de la libération de Maïa,je vais 3. retrouver un peu de chaleur amicale en assistant à l'unique représentation de mon

4. amie Sheila à l'olympia. Sachant les moments difficiles que je vivais ces derniers

5. jours, elle m'a appelé le week-end dernier pour me proposer de venir la revoir à

6. l'olympia.

7. C'est donc avec un vrai plaisir que j'irai l'applaudir dans cette salle mythique qu'est

8. l'olympia. 
9. J'irai voir l'amie, la fidèle présidente d'honneur d'Elus Locaux Contre le Sida et ce soir

10. surtout la grande chanteuse populaire qu'elle est depuis plus de 40 ans.

(21 septembre 2007)

Dans (1), la présence massive du pronom personnel et possessif de la première personne du singulier (7 occurrences, en italiques) indique qu'il s'agit d'un discours autocentré. L'auteur prend position tantôt par l'expression de ses émotions, tantôt par l'éloge de la chanteuse Sheila et de son concert. Les émotions présentes ou passées sont exprimées par les expressions «cette dure semaine» (ligne 2) et «les moments difficiles que je vivais» (1.4) dans lesquelles l'émotion éprouvée par l'auteur n'est pas explicitée mais connotée par les adjectifs décrivant ses journées précédentes : « dur et difficile $»^{2}$. Ces expressions signalent une prise de position intense, au sens où le politicien expose ses propres sentiments de chagrin et de frustration, voire d'indignation, face à un thème à la fois délicat et fondamental (cf. le billet de Jean-Luc Romero du 15 septembre 2007: "Je reste révolté qu'on doive quitter notre pays pour arrêter de souffrir quand il n'y a plus d'espoir de rémission »). A ces émotions négatives s'oppose la réaction positive à l'avenir que suscite le concert : « je vais retrouver un peu de chaleur amicale » (1. 2-3), « C'est avec un vrai plaisir que j'irai » (1. 7).

La prise de position dans ce billet est encore plus intense en ce qui concerne l'expression de l'axiologie positive envers le concert à venir et la chanteuse; il s'agit d'un discours apologétique. L'auteur évalue le concert par : «chaleur amicale » (1.3), "l'unique représentation" (l.3) et "l'applaudir dans cette salle mythique qu'est l'olympia» (1.7-8). Dans ces expressions, le substantif "chaleur», les adjectifs " amicale, unique » et «mythique » et le verbe "applaudir " (dans un sens figuratif) expriment l'affectivité en même temps qu'ils décrivent leurs dénotés : ils «énoncent, en même temps qu'une propriété de l'objet qu'ils déterminent, une réaction émotionnelle du sujet parlant en face de cet objet » (Kerbrat-Orecchioni 1997 [1980]: 84). Parallèlement, l'auteur exprime son affection et admiration à la chanteuse par : « mon amie » (1.3-4), "l'amie » (l.9), "la fidèle présidente» (1.9) et "la grande chanteuse populaire qu'elle est depuis plus de 40 ans » (1. 10). Le substantif « amie » est à la fois une description de l'objet et une évaluation affective qualifiant la relation entre l'auteur et la chanteuse ; les adjectifs « fidèle » et « grande » décrivent la personne mais expriment également l'estime; enfin, le syntagme " qu'elle est depuis plus de 40 ans » décrit la carrière tout en soulignant son importance. L'effet conjugué de ces expressions de prise de position tantôt face aux émotions de l'auteur, tantôt face au concert et à la chanteuse, témoigne d'une subjectivité élevée, voire intense, qui est monnaie courante dans un billet de blog de type journal extime.

Plantin (2011 : 191) constate que dans le discours politique en général, l'affichage des émotions est souvent un outil de l'ethos, de la construction d'une image crédible de soi, et cela est sans doute le cas dans (1) également. L'exemple (1) signale que les conventions tacites du genre en question n'appellent pas un tel affichage des émotions. Contrairement à (1), qui consiste en des émotions déclarées, les exemples suivants vont démontrer que le blog de politicien permet une grande variation dans le degré d'émotivité du discours.

19 Le billet (1) peut être également interprété comme un récit personnel dans une démarche continue de persuasion en faveur de la légalisation de l'euthanasie. Comme le montre Duranti (2006), les récits personnels sont employés dans les campagnes politiques de différentes façons pour construire une image crédible de la personne 
politique. Même si le présent récit (1) ne porte pas directement sur l'euthanasie, il expose l'adhésion de Romero à sa cause à travers l'émotivité par laquelle il réagit à la récente étape négative de sa lutte. Dans cette interprétation plus globale, (1) participe également de la notion d'engagement dans le sens de militer pour une cause politique ou sociale.

L'exemple (2) représente l'écriture polémique et constitue, par conséquent, un exemple univoque de la concordance partielle des notions de prise de position et d'engagement, car le billet représente une volonté de militer pour une cause dans la société. L'élément clé d'une polémique consiste, selon Amossy (2008), dans l'usage d'une tonalité agressive du langage au service de la confrontation de thèses. La tonalité agressive, pouvant aller jusqu'à la violence verbale, est définie par Amossy comme un discours argumentatif d'opposition qui enfreint d'une part les règles de la politesse - atteinte à la face de l'autre - et, d'autre part, les règles de la discussion rationnelle (2008: 99). Comme exemples de la violence verbale, on peut citer l'attaque directe - l'accusation, la dénomination dévalorisante, etc. -, les ressources du pathétique - la colère, la compassion envers la victime de l'opposant, etc. - et les ressources de l'humeur l'ironie, le sarcasme etc. (2008: 95).

21 L'exemple (2) est tiré du blog du Conseiller régional d'île de France et conseiller municipal de Persan, Emmanuel Maurel (PS, http://emmanuelmaurel.canalblog.com/). Il traite l'un des sujets politiques les plus fréquents dans les blogs des politiciens pendant le mois de septembre 2007 : l'amendement du recours aux tests ADN dans la vérification des liens familiaux des immigrés. Le billet dont (2) est extrait est écrit entièrement dans le même style polémique :

(2) (numéros de ligne rajoutés)

1. Il y aurait beaucoup à dire de la conception purement biologique de la famille

2. qu'implique un tel dispositif. Adoption, famille recomposée, etc.: dans les cerveaux

3. malades des promoteurs de l'amendement $\mathrm{ADN}$, les étrangers ne sont pas concernés par

4. ce genre de subtilités.

5. Mais il y a plus abject encore. On croyait les tests ADN réservés à la criminologie et à

6. la recherche scientifique et médicale. Des apprentis sorciers proposent désormais de

7. franchir une étape majeure : l'utilisation à des fins politiques. Comme dans « le meilleur

8. des mondes », comme dans « bienvenue à Gattaca », la génétique participerait du

9. contrôle social. Une fois la brêche ouverte, il n'y a plus de limites. Et

10. l'expérimentation sur les plus vulnérables précède souvent la généralisation à d'autres

11. catégories. Lorsque celles-ci se décident à réagir, il est trop tard. Autant que des salauds

12. Mariani et Hortefeux sont des irresponsables: ils mettent le doigt dans un engrenage

13. infernal.

(17 septembre 2007)

Dans (2), les pronoms de la première personne sont absents et par conséquent la subjectivité diffère de celle qui s'exprime dans (1): c'est la surabondance d'axiologiques, cette fois négatifs, qui constitue le degré élevé de subjectivité. La subjectivité forte est de nature agressive, et elle est employée pour s'opposer aux adversaires politiques - ce billet peut par conséquent être qualifié de polémique. Il 
porte atteinte à la face de l'adversaire et il enfreint également les règles de la discussion rationnelle. Par exemple, l'auteur se réfère aux personnes en charge de l'amendement par les expressions «les cerveaux malades des promoteurs de l'amendement $\operatorname{ADN} »(1.3)$, «des apprentis sorciers» (1.6), «salauds» (1.11) et «irresponsables» (1.12) qui expriment une évaluation subjective forte et négative de leur caractère. Sur le plan rhétorique, ces expressions sont considérées comme des arguments ad hominem car ils attaquent la personne au lieu de se concentrer sur le sujet du litige (cf. Amossy 2006: 139-140), ce qui transgresse les règles de la discussion rationnelle. De plus, l'auteur décrit les étrangers comme des victimes de l'amendement en les caractérisant par "les plus vulnérables » (1.10), et emploie ainsi un adjectif affectif-axiologique au superlatif. L'extrait (2) contient également une sous-estimation ironique «ce genre de subtilités » (1.4) qui se réfère aux formes de parenté autres que celle, biologique, auxquelles les immigrés n'auraient pas accès si l'amendement était adopté.

Ensuite, les références à l'amendement en soi expriment un jugement fort, voire une exagération. L'adjectif «abject» (1.5) et le syntagme "un engrenage infernal» (1.12-13) représentent des évaluatifs affectifs-axiologiques intenses. De plus, le scénario que l'auteur voit engendré par la possible adoption de l'amendement est décrit par des références intertextuelles dramatiques : il se réfère à un roman et à un film (Le meilleur des mondes d'Aldous Huxley [1931] et Bienvenue à Gattaca réalisé par Andrew Niccol [1997]), qui portent tous deux sur la thématique de l'eugénisme extrême sous-jacent à la manipulation du patrimoine génétique des fœtus humains. Ces scénarios sont rattachés à l'amendement actuel par l'avertissement « Une fois la brèche ouverte, il n'y a plus de limites» (1.9). En plus des arguments ad hominem décrits ci-dessus, cette description dramatique et hyperbolique de la situation causée par le parti opposé est, selon Koren (2003), typique de l'écriture polémique.

Une prise de position intense, telle qu'illustrée par (1) et (2), témoigne de l'aisance de l'expression de la subjectivité dans le genre du blog de politicien. Pourtant, si l'expression de la subjectivité est décomplexée, surtout dans les billets dits polémiques, elle ne l'est pas automatiquement dans les journaux extimes ou dans les autres types de blogs. Dans la partie qui suit, nous allons explorer des formes de subjectivité moins manifestes, et pourtant présentes.

\section{Subjectivité de degré intermédiaire}

L'un des cinq sous-genres repérés dans les blogs des politiciens est la réflexion. Elle se distingue de la polémique surtout par un degré moins élevé de subjectivité (cf. Lehti 2011: 1620-1624). Dans une réflexion, la personne politique présente l'analyse d'un sujet politique ou sociétal sans complètement cacher sa prise de position. Cependant, à la différence de la polémique, la subjectivité n'y est pas intense. L'exemple (3) du blog du sénateur du Finistère François Marc (PS, http://www.francois-marc.com/blog) est une réflexion sur l'état actuel de la subsistance des agriculteurs :

(3) (numéros de ligne rajoutés, caractères gras et soulignement (hyperlien) comme

dans l'original)

1. La course poursuite des prix agricoles

2. Beaucoup d'agriculteurs rencontrés ces derniers jours m'ont fait part de leurs

3. inquiétudes quant à la réduction de leur marge financière.

4. L'indice des prix agricoles révèle une importante progression des prix des 
matières

5. premières : + 0,4 \% pour le mois de juillet et $+3,9 \%$ par rapport à juillet 2006 .

6. Les prix de l'énergie augmentant à nouveau : $+0,8 \%$ sur un mois.

7. La principale augmentation concerne les aliments pour animaux qui continuent à

8. progresser, majoritairement ceux pour veaux $(+19,9 \%$ sur un an) et pour porcins

9. $(+14,1 \%)$. Les prix des engrais phosphatés renchérissent également $(+14,5 \%$ sur 10. un an).

11. Aujourd'hui les coûts de production agricoles grimpent jusqu'à étouffer les prix

12. rémunérateurs agricoles et limiter dangereusement les revenus agricoles.

13. Je considère que l'action revendicatrice des syndicats agricoles pour une juste marge,

14. indispensable à la survie du secteur, est dès lors très légitime en ce qui concerne les

15. « marges arrières ». Les agriculteurs doivent pouvoir vivre correctement de leur 16. travail...

(14 septembre 2007) subjectivité n'est pas intense, car au cœur du billet on trouve des expressions qui énoncent "une propriété objective, facilement vérifiable, du dénoté " (KerbratOrecchioni 1997 [1980] : 73), notamment les chiffres liés au développement des coûts de production agricole (1. 2-10). La prise de position, de degré intermédiaire, est manifeste dans les expressions suivantes : à la deuxième ligne, l'auteur expose son attachement à la cause en citant les discussions qu'il a menées avec les personnes concernées. Le quantifieur «beaucoup » (1.2) est relatif et exprime l'évaluation du sujet parlant; par l'usage de «beaucoup », l'auteur souligne l'importance du problème. De plus, l'auteur décrit et évalue l'état d'âme des agriculteurs par le substantif «inquiétudes» (1.3). Dans les lignes 11-12, l'auteur donne son évaluation de la situation: le verbe figuratif «étouffer» (1.11) et l'adverbe "dangereusement» (1.12) expriment une prise de position relativement intense. Finalement, les deux dernières lignes explicitent l'opinion de l'auteur. Le verbe d'opinion « considère » (l. 13), combiné au pronom de la première personne du singulier, est une indication explicite de la prise de position de l'auteur. Les adjectifs « juste» (l. 13), « indispensable» (l. 14) et « légitime» (1. 14) sont des évaluatifs axiologiques qui expriment des jugements de valeur. La déclaration à la fin du billet contenant le verbe modal « devoir » (Les agriculteurs doivent pouvoir vivre [...], 1.15-16) inclut une modalité déontique qui sert à «caractériser des jugements essentiellement prescriptifs qui [...] s'appuient sur des institutions (des systèmes de conventions) » (Gosselin 2010 : 361). Ainsi, l'auteur exige que les tiers indéfinis rendent possible ou assurent la possibilité aux agriculteurs de pourvoir à leur subsistance. De plus, l'adverbe "correctement» (1. 15) dans cette déclaration implique un jugement axiologique de ce qui est un niveau de vie souhaitable et normal, sans que l'auteur précise ce niveau.

Ce qui distingue (3) du billet polémique (2) est surtout le degré de subjectivité investi. Comme nous venons de le voir, (2) manifeste une prise de position intense alors que le pivot de (3) est l'énumération des faits. Même si (3) ne prétend pas à la neutralité, le degré de subjectivité y est moins élevé que dans (2). De plus, l'élément d'attaque contre un opposant ou un autre discours est absent dans (3) : il s'agit d'une simple expression de protestation contre l'état des choses actuel. 
La subjectivité du politicien auteur d'un blog est naturellement présente dans tous les billets étiquetés comme journal extime car ils sont liés à sa personne et ses activités. Ces textes sont caractérisés par un usage fréquent des pronoms de la première personne indiquant non seulement la subjectivité déictique mais également l'expression des expériences de l'auteur. Pourtant, l'intensité de la prise de position varie considérablement dans cette catégorie, comme nous le montre (4), en comparaison à l'affectivité de (1) dans la sous-section précédente. L'exemple (4) est tiré du blog du maire de Grenoble Michel Destot (PS, le site n'existe plus) :

(4) (numéros de ligne rajoutés)

1. Rencontre avec les habitants de Saint-Laurent

2. J'ai évidemment repris depuis le début du mois de septembre le rythme hebdomadaire

3. de mes rencontres avec les habitants dans les différents quartiers de Grenoble. Après

4. Mistral et la Villeneuve, j'étais aujourd'hui en fin d'après-midi dans le quartier

5. Saint-Laurent.

6. L'occasion évidemment de faire le point sur les principaux chantiers de rénovation des

7. immeubles de la rue Saint-Laurent mais aussi d'échanger sur notre volonté de

8. réaménager les berges de l'Isère. Une volonté qui s'inscrit dans le cadre du projet

9. Cœur de Ville, Cœur d'Agglo dont la mise en œuvre se poursuit actuellement avec

10. la mise en accessibilité du Jardin de Ville et l'aménagement de la gare basse du

11. Téléphérique.

(20 septembre 2007)

Le billet (4) contient plusieurs expressions déictiques (je, mes, notre, aujourd'hui, actuellement) qui lient le texte au moment de l'énonciation, à la personne politique et à la communauté. Même si les pronoms de la première personne rendent le discours autocentré, la subjectivité déictique est considérée par Kerbrat-Orecchioni (1997 [1980] : 149) comme étant « objective ", dans la mesure où les expressions déictiques (temporelles, spatiales, personnelles) ne font pas l'objet d'un jugement évaluatif. Malgré l'objectivité des déictiques, Charaudeau (1992: 158-159) remarque que le pronom «nous » est employé fréquemment dans le discours politique d'une manière stratégique. Dans le cas de (4), le pronom possessif «notre » (1. 7) réfère aux acteurs de la politique municipale dont le maire est le porte-parole.

En plus de la subjectivité déictique dans (4), l'auteur exprime une prise de position positive vis-à-vis des projets présentés lors de la rencontre. Cette prise de position se transmet par l'usage du substantif « volonté » (1. 7 et 8) qui signifie «[c]e que souhaite, ce que désire une collectivité, un groupe " (Larousse en ligne, s. v. "volonté ») et comporte ainsi une évaluation positive à la fois du processus de réaménagement et de l'objet de celui-ci, la zone des berges de l'Isère. En plus de cette évaluation positive, la subjectivité de l'auteur est exprimée par l'adverbe " évidemment » (1. 2 et 6). Il est une indication des modalités épistémiques, modalités que Gosselin (2010:325) appelle des "vérités subjectives", dans la mesure où elles "ne renvoient pas à une réalité indépendante des sujets qui la considèrent, mais à l'évaluation subjective de cette réalité ». Ainsi, l'adverbe " évidemment » dans (4) exprime une évaluation subjective de l'évidence de la prédication de la première phrase ( «J'ai repris depuis le début du mois de septembre le rythme hebdomadaire de mes rencontres») et du substantif «l'occasion». 
31 L'effet conjugué des expressions subjectives analysées est celui d'une intensité peu élevée: la prise de position n'est guère intense dans (4). De plus, même si (4) est marquée par la perspective subjective du récit personnel de la rencontre avec les habitants et de la mise en avant des réalisations de sa communauté, l'auteur est présenté uniquement dans son rôle de maire : il informe le lecteur de ses activités professionnelles et des réalisations de sa communauté, au lieu de dévoiler sa vie privée ou ses propres expériences et émotions comme dans (1). En outre, les opinions politiques de l'auteur ne sont pas accentuées.

Quant aux autres types, le sous-genre du tableau d'affichage, fonctionnant comme un recueil d'annonces ou publicités, accueille un éventail de sujets et de manières de dire. Parmi ces billets, nous nous concentrons sur ceux qui se focalisent sur l'acte perlocutionnaire du lecteur. L'exemple (5), du blog national ${ }^{3}$ du sénateur de l'Orne Alain Lambert (http://www.alain-lambert-blog.org/) en est un exemple. Parallèlement à (2), le billet traite de l'amendement de loi sur l'imposition des tests ADN aux immigrés afin de vérifier les liens familiaux. Si la plupart des billets portant sur cette thématique dans notre corpus expriment une opposition fervente, (5) offre un exemple d'écriture qui semble objective mais représente pourtant une prise de position claire :

(5) (numéros de ligne rajoutés)

1. Immigration : le recours aux tests ADN

2. L'annonce de l'amendement au projet de loi sur l'immigration, adopté par la commission

3. des lois de l'Assemblée Nationale, a lancé une vive polémique. Il prévoit le recours aux

4. tests ADN pour les futurs candidats au regroupement familial dans le but de restreindre

5. l'immigration mais aussi de rendre impossible la possession de faux papiers.

6. Plusieurs questions se posent alors:

7. Cet amendement vous semble-t-il opportun?

8. D'après vous, quels en sont les avantages et les inconvénients?

9. Et aussi peut-on penser l'immigration en dehors de l'Union européenne et de l'espace

10. Schengen?

11. Avant de contribuer au débat par le biais de vos commentaires, que j'espère nombreux,

12. vous êtes invités à visionner une vidéo sur ce sujet en cliquant ici !

13. Le sujet est très sérieux et surtout délicat, c'est pourquoi il m'a semblé utile d'en

faire le

14. sujet d'un débat digne entre nous. En sages, comme d'habitude.

(18 septembre 2007)

Parmi les 25 bloggeurs qui représentent l'UMP dans notre corpus, Lambert est l'un des deux qui parlent de cet amendement controversé proposé par leur parti; alors que parmi les 43 bloggeurs socialistes et les 8 bloggeurs de l'UDF-Modem, ce sujet est abordé par 12 auteurs (écrivant au total 14 billets sur le sujet) qui, sans exception, expriment leur opposition. Dans (5), Lambert ne révèle pas son opinion sur l'amendement; au lieu de cela, il invite les lecteurs à exprimer la leur. Cet acte illocutoire de requête (d'avis) est une manifestation explicite de l'intersubjectivité, soit de la prise en compte du destinataire.

L'intensité de la prise de position dans (5) n'est pourtant pas à juger à l'aune de l'expression de l'adhésion au propos référentiel de l'amendement ou de la politique d'immigration en général, mais à celle du débat en soi, initié par les questions directes 
des lignes 7-10. À ce propos, la prise de position est exprimée par l'expression «que j'espère nombreux » (l.11), où le verbe " espérer " indique une modalité boulique qui exprime « des désirs, des volontés, des souhaits » (Gosselin 2010 : 351). Dans (5), l'espoir est attaché au nombre futur des commentaires. Un autre marqueur de prise de position est le point d'exclamation (1.12) qui renforce l'invitation adressée au lecteur. De plus, les expressions évaluatives positives "débat digne entre nous " (1.14) et «en sages " (1.14) expriment l'adhésion de l'auteur à ce débat, en même temps qu'elles transmettent la politesse langagière par l'attention accordée au lecteur et par son souhait que personne ne soit insulté dans le futur débat. Finalement, aux lignes 11-14, l'auteur emploie des pronoms de la première personne du singulier et du pluriel et de la deuxième personne du pluriel. Le «nous » ici inclut le lecteur, il est employé pour appeler celui-ci à se solidariser (cf. Charaudeau 1992: 159). Le degré de la prise de position exprimé dans ce billet - surtout par le biais de l'intersubjectivité - peut être caractérisé comme intermédiaire: la subjectivité est clairement présente sans être pour autant le pivot du texte.

En plus des invitations à l'action, le sous-genre du tableau d'affichage contient des annonces courtes de sujets variés qui manifestent également un degré de subjectivité investi intermédiaire, presque faible. L'exemple (6) du blog du conseiller municipal du Havre Marc Migraine (UDF-MoDem, http://www.auhavre.com/blog2/) représente ce type :

(6) (numéros de ligne et italiques rajoutés)

\section{1. tout baigne}

2. A fin août, le trafic cumulé du port du Havre s'établit à 52,8 millions de tonnes

3. (avitaillement inclus). soit une hausse de $8,3 \%$.

4. L'activité conteneurs reste très bien orientée, sur les huit premiers mois de l'année 17,4

5. Mt de tonnes ont été traités. Les vracs solides progressent de $27,8 \%$, à $3 \mathrm{Mt}$; le trafic

6. des vracs liquides reste stable à 30,9 Mt à fin août 2007. Le trafic transmanche entre

7. Le Havre, Portsmouth et Newhaven est en hausse : 262000 passagers transportés entre

8. janvier et août $2007(+40,1 \%)$.

9. d'autre part les travaux concernant les six nouveaux postes à quai de Port 2000 ont

10. démarré et les installations devraient être livrées entre 2009 et 2010 avec un début

11. d'exploitation en 2011 après la mise en place des équipements.

(11 septembre 2007)

Ce billet (6) est caractérisé par l'énumération des faits, notamment des chiffres, pourcentages et dates concernant le trafic et les constructions du port du Havre. Ce qui distingue (6) du sous-genre de la réflexion (exemple 3) est la brièveté du billet et l'absence de toute opinion de l'auteur. Une prise de position positive, voire promotrice, est néanmoins repérable dans le texte. Premièrement, le titre "tout baigne " (l.1) exprime une évaluation positive de la situation. Ensuite, l'expression évaluative «très bien orientée » (1.4) marque le contentement de l'auteur vis-à-vis des activités portuaires. Troisièmement, le verbe auxiliaire devoir au conditionnel « devraient être livrées » (1.10) indique non seulement une modalité épistémique sur la probabilité de la réalisation des installations basée sur le savoir de l'auteur, mais également, dans ce contexte, une modalité appréciative, car il inclut un effet «de mise en perspective 
éventuelle» (Charaudeau 1992 : 473) qui exprime une hypothèse souhaitée. En outre, il est nécessaire de mentionner que le registre informel du titre (1.1) rajoute un léger élément humoristique au billet en le distinguant ainsi d'un style complètement neutre et objectif.

\section{Subjectivité faible}

Cette dernière sous-section de l'analyse se concentre sur des billets où le langage laisse voir un taux faible de subjectivité. Cette écriture «neutre » est surtout de mise dans les billets nommés vitrines qui publient des documents initialement parus ailleurs, tels que communiqués de presse, discours prononcés, articles de journaux ou émissions de télévision (à travers des hyperliens ou en format de vidéoclips). Dans les termes de Charaudeau (1992 : 649-650), dans ces cas « le Propos est un Texte déjà produit par un autre locuteur, et le sujet parlant n'aurait donc à jouer qu'un rôle de rapporteur (dont on sait qu'en réalité il peut être plus ou moins objectif)». Dans les billets vitrines, la prise de position des politiciens est souvent faible au texte qu'ils présentent.

Les textes publiés sont souvent introduits d'une façon brève, comme dans (7), qui est tiré du blog du conseiller municipal de Vertou Laurent Dejoie (UMP, http:// www.laurentdejoie.com/) et dans (8), du blog du conseiller municipal du Plessis Robinson Benoît Marquaille (PS, http://www.benoitmarquaille.blogs.com/) :

(7)

\section{SARKOZY à NANTES}

Nicolas SARKOZY, après avoir rendu hommage à Guy MÔQUET à Chateaubriant, a prononcé un discours sur la fonction publique à l'Institut Régional d'Administration de NANTES. Vous pouvez lire, écouter ou voir son discours intégral. (liquez ici) (19 septembre 2007)

(8)

Les élus socialistes au Conseil général demandent un " Vélib’ " pour le 92

Les élus socialistes ont interpellé le président du Conseil Général pour que soit rapidement lancée la consultation d'appel d'offres pour doter notre département d'un réseau de location de vélos similaire à celui existant pour la ville de Paris.

Voir le communiqué de presse sur le site du groupe socialiste

(17 septembre 2007)

L'auteur de (7) donne le lien vers le discours de Sarkozy et dans (8), il s'agit d'un communiqué de presse. Les introductions à ces liens sont relativement neutres, malgré la conjonction «pour que » (8) qui exprime un but envisagé, et par conséquent la prise de position de l'auteur. De plus, à la fin des billets (7) et (8) respectivement, les auteurs inscrivent le destinataire dans le langage par les actes directifs d'offre (d'hyperliens), ce qui témoigne de l'intersubjectivité; dans (7), le pronom "vous » est affiché, alors que dans (8) l'acte est réalisé par un infinitif.

Il est toutefois important de souligner que, comme le note Myers (2010: 95), même la publication d'une simple liste d'hyperliens dans un blog implique un positionnement, dans la mesure où elle dévoile les préférences et intérêts de l'auteur. Mais, si le choix d'aborder sur l'agora numérique le discours de Sarkozy ou le communiqué de presse des élus socialistes est subjectif, ce qui est en jeu ici est plutôt la notion d'engagement que celle de la prise de position. Les propos référentiels des deux billets sont politiques et leur affichage témoigne d'une volonté de militer avec les siens pour des causes partagées. 
41 Nous considérons néanmoins les blogs vitrines tels que (7) et (8) comme des exemples d'une prise de position faible, car même si le choix du document présenté est subjectif et l'intersubjectivité explicite, le langage en soi contient peu de marqueurs de prise de position. L'auteur n'exprime pas d'émotion ou d'évaluation envers le document qu'il présente. Il nous semble par conséquent qu'un blog vitrine est un bulletin relativement neutre et que dans ce sous-genre, la prise de position de l'auteur est moins importante que la simple diffusion de l'information.

\section{Conclusion}

L'analyse proposée dans cet article concernant la prise de position dans les blogs des politiciens illustre un continuum du langage allant du pôle «subjectif » vers le pôle «neutre ». Ce continuum suggère que ce genre permet de nombreux degrés et modes d'inscription de la subjectivité dans le discours. Contrairement à l'idée générale qu'on se fait $\mathrm{du}$ format blog comme le lieu d'une exposition franche et ouverte de la subjectivité, le genre du blog de politicien ne manifeste pas nécessairement de la subjectivité : si le langage des billets du type polémique et de certains billets du type journal extime témoigne d'un degré fort de subjectivité investi, le tableau d'affichage, certains billets $\mathrm{du}$ type journal extime et la réflexion manifestent un degré intermédiaire de prise de position - et, parmi ces trois, le tableau d'affichage est surtout marqué par l'intersubjectivité. Finalement, les blogs vitrines révèlent un taux faible de subjectivité : la prise de position est peu inscrite dans le langage.

La variation dans l'expression de la prise de position atteste de l'hétérogénéité inhérente au genre du blog de politicien. Ce genre ne semble pas imposer de conventions tacites en ce qui concerne les modes et le degré de subjectivité investi : il permet une variation considérable. Le genre est également hybride ; comme le montre la dénomination des sous-genres, c'est un lieu par excellence de mélange de genres. Qui plus est, le blog de politicien est un lieu où s'interpénètrent de nombreux types de discours (discours politique, discours médiatique, etc.), champs discursifs (discours socialiste, discours républicain, etc.) et registres communicationnels (discours polémique, discours apologétique, etc.). Toute cette hétérogénéité configure un genre dans lequel la similarité d'un texte à l'autre se trouve plus dans les facteurs contextuels (format, participants) que dans le matériau langagier. Cette hétérogénéité permet également l'usage du genre à des buts persuasifs variés; en ce qui concerne la construction d'une image crédible de l'auteur, le présent article démontre que l'ethos créé peut varier d'un polémiqueur engagé à un médiateur neutre.

Le corpus de l'étude décrit un moment dans l'histoire d'un genre émergent, les blogs de politiciens français pendant le mois de septembre 2007. Ce travail offre une possibilité de comparaison diachronique ; l'expression de la subjectivité de ce corpus pourrait être comparée à celle d'un corpus plus récent. En 2007, le blog était l'une des premières formes du média social adoptées par les politiciens, alors qu'aujourd'hui, en 2012, de nombreux politiciens emploient également Twitter et Facebook, par exemple. Il serait intéressant d'examiner si l'arrivée de ceux-ci a eu une influence sur les blogs, et d'explorer selon quelles modalités les politiciens gèrent leur identité numérique à travers les différents genres et formats. 


\section{BIBLIOGRAPHIE}

Adam, Jean-Michel. 1997. « Unités rédactionnelles et genres discursifs : cadre général pour une approche de la presse écrite », Pratiques, 94, 3-18

Amossy, Ruth. 2006. L'argumentation dans le discours (Paris : Colin)

Amossy, Ruth. 2008. « Modalités argumentatives et registres discursifs : le cas du polémique ", Gaudin-Bordes, Lucile \& Geneviève Salvan (éds). Les registres : Enjeux stylistiques et visées pragmatiques (Louvain-la-Neuve : Bruylant-Academia)

Amossy, Ruth \& Roselyne Koren. 2004. « Présentation », Semen, 17

Benveniste, Émile. 1966. Problèmes de la linguistique générale (Paris : Gallimard)

Charaudeau, Patrick. 1992. Grammaire du sens et de l'expression (Paris : Hachette)

Cohen-Wiesenfeld, Sivane. 2004. « L'inscription de la subjectivité dans le discours diplomatique », Semen, 17

Duranti, Alessandro. 2006. « Narrating the political self in a campaign for U.S. Congress », Language in Society, 35 (4), 467-497

Gosselin, Laurent. 2010. Les modalités en français. La validation des représentations (Amsterdam, New York : Rodopi)

Grosse, Ernst-Ulrich. 2001. « Evolution et typologie des genres journalistiques », Semen, 13

Hancil, Sylvie. 2011. « Avant-propos ». Hancil, Sylvie (éd.). Marqueurs discursifs et subjectivité (Mont-Saint-Aignan : Publications des universités de Rouen et du Havre), 7-11

Herman, Thierry \& Nicole Jufer. 2001. «L'éditorial, “vitrine idéologique du journal” ?», Semen, 13

Herring, Susan C. \& John C. Paolillo. 2006. « Gender and genre variation in weblogs », Journal of Sociolinguistics, 10 (4), 439-459

Janoschka, Anja. 2010. « Direct e-communication : Linguistic weapons in a political weblog ». Okulska, Urszula \& Piotr Cap (éds). Perspectives in Politics and Discourse (Amsterdam : John Benjamins), 215-236

Jereczek-Lipińska, Joanna. 2007. « Le blog en politique - outil de démocratie électronique participative? ", Glottopol, 10, 162-172

Kerbrat-Orecchioni, Catherine. 1997 [1980]. L'énonciation : De la subjectivité dans le langage (Paris : Colin)

Koren, Roselyne. 2003. «Stratégies et enjeux de la "Dépolitisation par le langage" dans un corpus de presse actuelle ", Recherches en Communication, 20, 65-83

Koren, Roselyne. 2004. «Argumentation, enjeux et pratique de l'“engagement neutre” : le cas de l'écriture de presse », Semen, 17

Koren, Roselyne. 2009. "De la parole "représentative" à la parole "participative" : le journalisme politique revisité par La gazette d'@rrêt sur images », présentation au colloque Le français parlé dans les medias, Université de Lausanne, 1-4 septembre 2009

Larousse dictionnaire, http://www.larousse.com/en/dictionaries/french/ 
Lehti, Lotta. 2011. « Blogging politics in various ways : A typology of French politicians' blogs ", Journal of Pragmatics, 43 (6), 1610-1627

Miller, Carolyn R. 1984. « Genre as social action », Quarterly Journal of Speech, 70, 151-167

Miller, Carolyn R. \& Dawn Shepherd. 2004. « Blogging as Social Action : A Genre Analysis of the Weblog », Gurak, Laura, Smiljana Antonijevic, Laurie Johnson, Clancy Ratliff \& Jessica Reyman (éds). Into the Blogosphere : Rhetoric, Community, and Culture of Weblogs (en ligne)

Miller, Carolyn R. \& Dawn Shepherd. 2009. «Questions for genre theory from the blogosphere », Giltrow, Janet \& Dieter Stein (éds.). Genres in the Internet (Amsterdam : John Benjamins), 263-290

Myers, Greg. 2010. Discourse of Blogs and Wikis (London : Continuum)

Plantin, Christian. 2011. Les bonnes raisons des émotions. Principes et méthode pour l'étude du discours émotionné (Bern : Peter Lang)

Suomela-Salmi, Eija. 2009. «La construction de soi dans les blogs des politiciens français », Havu, Eva, Mervi Helkkula \& Ulla Tuomarla (éds). Du côté des langues romanes : Mélanges en l'honneur de Juhani Härmä (Helsinki : Société Néophilologique), 265-281

Tisseron, Serge. 2011. « Intimité et extimité », Communications, 88, 83-91

\section{NOTES}

1. Ces situations varient par exemple d'une personne publiant ses tenues du jour (http:// www.estelleblogmode.com/) à un comité d'organisation d'un colloque scientifique offrant des informations sur l'évènement (http://blogs.helsinki.fi/dialog3/).

2. Jean-Luc Romero, président de l'Association pour le Droit de Mourir dans la Dignité (ADMD) militant pour la légalisation de l'euthanasie active, se réfère ici au décès de l'actrice française Maïa Simon. La comédienne, atteinte d'un cancer incurable, a voyagé en Suisse pour avoir recours à un « suicide médicalement assisté » car la législation française l'interdit.

3. En 2007, Alain Lambert avait deux blogs : l'un appelé «national » et l'autre étiqueté comme « régional » (le site n'existe plus). Ce dernier était consacré aux sujets liés à la circonscription de l'auteur, le département de l'Orne.

\section{RÉSUMÉS}

Le blog de politicien est un genre discursif hybride dont les conventions tacites permettent une large variation en ce qui concerne les sujets et les manières d'écrire. L'article examine l'inscription de la subjectivité dans le langage des billets de blog écrits par des politiciens, afin de présenter les différents modes et degrés de manifestation de la subjectivité dans ce genre. L'analyse d'un corpus de 80 blogs de politiciens français, étudié à travers des exemples prototypiques des cinq sous-genres repérés au sein du genre, démontre que les billets constituent un continuum allant du pôle "subjectif » vers le pôle «neutre", et que le genre permet aussi bien une affectivité et des polémiques intenses qu'une objectivité de médiateur et l'effacement de la prise de position de l'auteur. 
Politician's blog is a hybrid genre where tacit conventions allow a large variety of topics and writing styles. The article examines the marking of subjectivity in the language of the politicians' blog posts, in order to present the ways and degrees in which subjectivity is manifested in the genre. The analysis of a material of 80 French politicians' blogs, examined through prototypical examples of five sub-genres identified in the material, reveals that the posts form a continuum from the "subjective" pole towards the "objective" one, and that the genre allows the expression of intense affect and fierce polemics as well as objective mediation and the concealment of the author's opinions.

\section{INDEX}

Mots-clés : blog de politicien, discours politique, ethos, genre, subjectivité dans le langage

Keywords : ethos, genre, political discourse, politicians' blog, subjectivity in language

\section{AUTEUR}

\section{LOTTA LEHTI}

Université de Turku 